\title{
Sequence variation in the mitochondrial DNA control region of wild African cheetahs (Acinonyx jubatus)
}

\author{
ABIGAIL R. FREEMAN $\dagger$, DAVID E. MACHUGH $\$$, SEÁN MCKEOWN**§, \\ CHRISTIAN WALZER $\uparrow$, DAVID J. MCCONNELL $\uparrow \S \&$ DANIEL G. BRADLEY*† \\ $\dagger$ Department of Genetics, Smurfit Institute of Genetics, Trinity College, Dublin 2, Ireland, $₫$ Department of Animal \\ Science and Production and Conway Institute of Biomolecular and Biomedical Research, Faculty of Agriculture, \\ University College Dublin, Belfield, Dublin 4, Ireland, §Fota Wildlife Park, Carraigtwohill, County Cork, Ireland and \\ - Salzburger Tiergarten Hellbrunn, A-5081 Anif, Austria
}

Five hundred and twenty-five bp of mitochondrial control region were sequenced and analysed for 20 Acinonyx jubatus and one Felis catus. These sequences were compared with published sequences from another domestic cat, 20 ocelots (Leopardus pardalus) and 11 margays (Leopardus weidii). The intraspecific population divergence in cheetahs was found to be less than in the other cats. However variation was present and distinct groups of cheetahs were discernible. The $80 \mathrm{bp}$ RS2 repetitive sequence motif previously described in other felids was found in four copies in cheetah. The repeat units probably have the ability to form secondary structure and may have some function in the regulation of control region replication. The two central repeat units in cheetah show homogenization that may have arisen by convergent evolution.

Keywords: Acinonyx jubatus, control region, Felidae, genetic diversity, mitochondrial DNA, tandem repeats.

\section{Introduction}

The cheetah (Acinonyx jubatus) is a highly specialized member of the Felidae and is renowned as the fastest land animal. Its survival in the wild is threatened throughout its habitat with a current population estimate of only 15000 wild individuals. Five subspecies of cheetah have been classified. The Southern African subspecies, Acinonyx jubatus jubatus, is found in Namibia, Botswana, Zimbabwe and South Africa. The other four subspecies are: $A . j$. raineyi which is found in Kenya, Uganda and Tanzania; A. j. soemmeringi from Sudan, Ethiopia, Chad, Central African Republic, Northern Nigeria and Niger; A. j. hecki from Southern Mauritania, Senegal, Burkina Faso, southern Mali and northern Benin and A. j. venaticus from Iran and Egypt (O'Brien et al., 1986; Nowell \& Jackson, 1996).

The lack of genetic diversity in A. j. jubatus was originally documented in studies using allozymes (O’Brien et al., 1983, 1985), spermatozoan morphology

\footnotetext{
*Correspondence. E-mail: dbradley@tcd.ie

**Present address: H.E. Sheikh Butti Bin Juma al Maktoum's Wildlife Centre, PO Box 7237, Dubai, United Arab Emirates.
}

(O'Brien et al., 1983, 1985, 1986), fluctuating asymmetry (Wayne et al., 1986) and skin graft analysis (O'Brien et al., 1985, 1986). The amount of information that these physical measurements can reveal about a lack of diversity at the genetic level has been questioned (Caughley, 1994). However, the physical effects reported to be as a result of inbreeding depression in the cheetah in these papers are typical of those in inbred laboratory mice (O'Brien et al., 1985). Samples from both $A$. $j$. jubatus and $A . j$. raineyi were included in subsequent studies. A larger panel of allozymes revealed that the genetic distance between the two subspecies was 0.004 which is eight times less than the genetic distance among disparate human groups (O'Brien et al., 1987).

More recently, minisatellites, mitochondrial RFLPs (Menotti-Raymond \& O'Brien, 1993) and microsatellites (Menotti-Raymond \& O'Brien, 1995) have been used to look at cheetah genetic diversity. Differences between these two subspecies were detectable and the levels of genetic diversity were not as low as previous studies might have suggested. However, microsatellite variation was lower in cheetah than in any other felid species examined (Menotti-Raymond \& O'Brien, 1995). Mitochondrial RFLPs revealed several mitotypes for 
each subspecies. The A. j. jubatus samples appeared to be more diverse and suggested that the northern subspecies was derived from its southern counterpart or had lost diversity subsequent to the separation of the subspecies. The close relationship between two subspecies was examined and implied that an ancient bottleneck may have occurred before they diverged (Menotti-Raymond \& O'Brien, 1993). The range of the cheetah may have undergone a dramatic contraction at the end of the Pleistocene era, a time when a large number of species became extinct (O'Brien et al., 1987). Several smaller bottlenecks may also have occurred since then. This is supported by the molecular data which dates a putative bottleneck at approximately 10000 years ago (Menotti-Raymond \& O’Brien, 1993).

Mitochondrial DNA (mtDNA) evolves more rapidly than nuclear single-copy DNA and the control region is the fastest evolving section of the mitochondrial genome. Its sequence diversity has been widely exploited in population studies (Brown et al., 1986). Here we use the mitochondrial control region to assess the genetic diversity of three subspecies of cheetah. We intend to examine the relationship among the three subspecies and contrast cheetah mtDNA diversity with that found in three other felid species.

Five different types of repeat arrays have been characterized in mitochondrial control regions and have been denoted RS1-RS5 (Hoelzel et al., 1993a, 1994). The domestic cat and many other carnivores contain the RS2 and RS3 repeat units (Lopez et al., 1996). Expansion and contraction of these arrays can lead to heteroplasmy and length variation in the molecule (Hoelzel et al., 1993b). It has been found that sequence homogenization can occur in the central repeats by concerted evolution; the tendency for the elements of an array to become homogenized by reiterated rounds of duplication and deletion of repeats (Solignac et al., 1986).

\section{Materials and methods}

\section{Sample collection}

Seventeen of the cheetah blood samples were obtained from wild Namibian Acinonyx jubatus jubatus (Aju-01Aju-17). One of the blood samples came from a hybrid captive animal with a raineyi maternal lineage from Vienna Zoological Gardens and two of the samples are from animals that were bought from a market in Somalia, having been captured from the wild in Northern Africa and belonging to the subspecies A. j. soemmeringi (Aju-29 and Aju-30). A domestic cat blood sample was obtained from a local veterinarian. Cheetah blood samples had been stored in $\mathrm{K}_{3}$-EDTA (15\%)-treated tubes at $4^{\circ} \mathrm{C}$ for 5 years. DNA was extracted from the samples and subsequently purified using proteinase $\mathrm{K} /$ sodium dodecyl sulphate (SDS) and phenol/chloroform/isoamyl alcohol (Sambrook et al., 1989).

Extracted DNA was then further purified using the Boehringer Mannheim High Pure PCR purification kit (Cat. no. 1732668). The DNA clean-up was carried out according to the manufacturer's instructions except for that the washing buffer was made up with $80 \%$ isopropanol instead of $80 \%$ ethanol. Negative controls were done in parallel with all of the extraction and purification steps.

\section{PCR amplification and DNA sequencing}

Primers were designed using the domestic cat whole mitochondrial genome (Genbank U20753). Both strands were amplified using one biotinylated and one nonbiotinylated primer. The two primers used were: CHF3: $3^{\prime}$ CTC CCT AAG ACT TCA AGG AAG $5^{\prime}$ and CHR3: 3' CAT CTG GTT CTT ACT TCA GG 5'. These were designed to amplify a section of the control region located between tRNA PROLINE and the conserved area in the middle of the control region.

The PCR reaction volume was $50 \mu \mathrm{L}$ consisting of dNTPs $(200 \mu \mathrm{M}), 15 \mathrm{~mm}$ PCR buffer: $500 \mathrm{~mm} \mathrm{KCl}$, $100 \mathrm{~mm}$ Tris- $\mathrm{HCl}, \mathrm{pH} 9.0,15 \mathrm{~mm} \mathrm{MgCl}_{2}, 4$ units $\mathrm{Taq}$ DNA Polymerase, forward primer $(4.0 \mu \mathrm{M})$ and reverse primer $(4.0 \mu \mathrm{M})$. The reaction was carried out in an MJ Research Peltier Thermal Cycler-200 DNA Engine for 40 cycles of $40 \mathrm{~s}$ at $94^{\circ} \mathrm{C}, 40 \mathrm{~s}$ at $59^{\circ} \mathrm{C}$ and $1 \mathrm{~min} 30 \mathrm{~s}$ at $72^{\circ} \mathrm{C}$. Success of the PCR reaction was confirmed by visualizing the reaction products alongside negative controls on a $1 \%$ agarose gel.

Biotin-labelled PCR products were purified using Dynabeads (Dynal A.S.) according to the manufacturer's instructions. Standard $\alpha-{ }^{35}$ S ATP Sequenase ${ }^{\circledR}$ chemistry was used for the sequencing reactions from single-stranded template from 17 of the samples. Three

Fig. 1 Reduced Clustal w alignment of part of the cheetah and domestic cat control regions (including RS2) showing only variable nucleotide positions. The nine cheetah haplotypes identified are designated Aju and the two domestic cats are abbreviated to Fca. The Fca-01 sequence is taken from Genbank (Accession number U20753). A dash (-) indicates a deletion compared to the consensus (Aju-01). The sequences are numbered in relation to Fca-01 and run from nucleotide positions 16310-16834 in this sequence. Roman numerals have been used to designate insertions in the cheetah sequence relative to Fca-01. The first two digits of the nucleotide positions have been left out for simplification. 
Aju-01
Aju-03
Aju-08
Aju-12
Aju-16
Aju-17
Aju-23
Aju-29
Aju-30
Fca-01
Fca-02

Aju-01

Aju-03

Aju-08

Aju-12

Aju-16

Aju-17

Aju-23

Aju-29

Aju-30

Fca-01

Fca-02

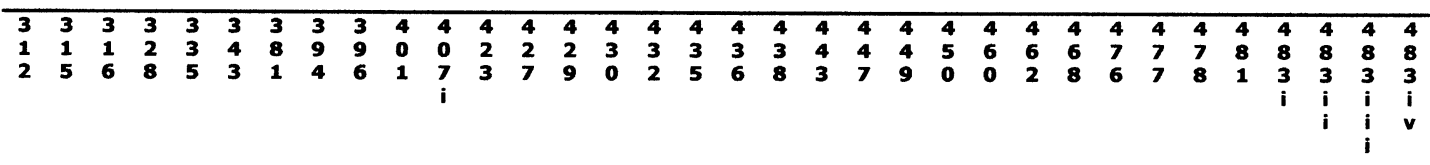

C C T C T C C C C A C T T T T CACT G T T G TACC C G T A T A A 
of the samples were sequenced commercially using an ABI PRISM sequencer, model 377.

\section{Sequence analysis}

Statistical analysis of sequence data was performed using the ARLEQUIN package (version 1.1, Schneider et al., 1997). Alignments and bootstrapping were carried out using CLUSTAL W and Kimura's 2-parameter model correcting for multiple substitutions (Kimura, 1980; Thompson et al., 1994). The PHYLIP package (version $3.572 \mathrm{c}$, Felsenstein, 1993) was used to construct distance matrices and phylogenetic trees. The median network was constructed according to the method outlined by Bandelt et al. (1995). MFOLD from The Wisconsin Package version 9.1, Genetics Computer Group (GCG), Madison, Wisconsin was used to determine potential sequence secondary structure. The sequence data for the margays and ocelots was obtained from: http://rex.nci.nih.gov/RESEARCH/basic/lgd/data/eizirik98_12.htm (Eizirik et al., 1988).

\section{Results}

\section{Variation in the cheetah mitochondrial control region}

Five hundred and twenty-five bp pairs (corresponding to 16310-16834 in Fca-01) were sequenced from each of 20 cheetah samples and one domestic cat sample (Fig. 1). Twenty-seven polymorphic sites were identified in the cheetah sequences all of which revealed transition substitutions. A reduced median network (Fig. 2) illustrates phylogenetic relationships among the cheetah haplotypes (Bandelt et al., 1995). All of the samples that cluster on the left-hand side of the network came from wild Namibian animals and belong to the Southern African A. j. jubatus subspecies. The animals on the right hand side of the network are from two different subspecies; $A$. $j$. soemmering $i$ and $A$. $j$. raineyi which are native to North and Central Africa. Each half of the network is further split into two clades differing by at least eight substitutions.

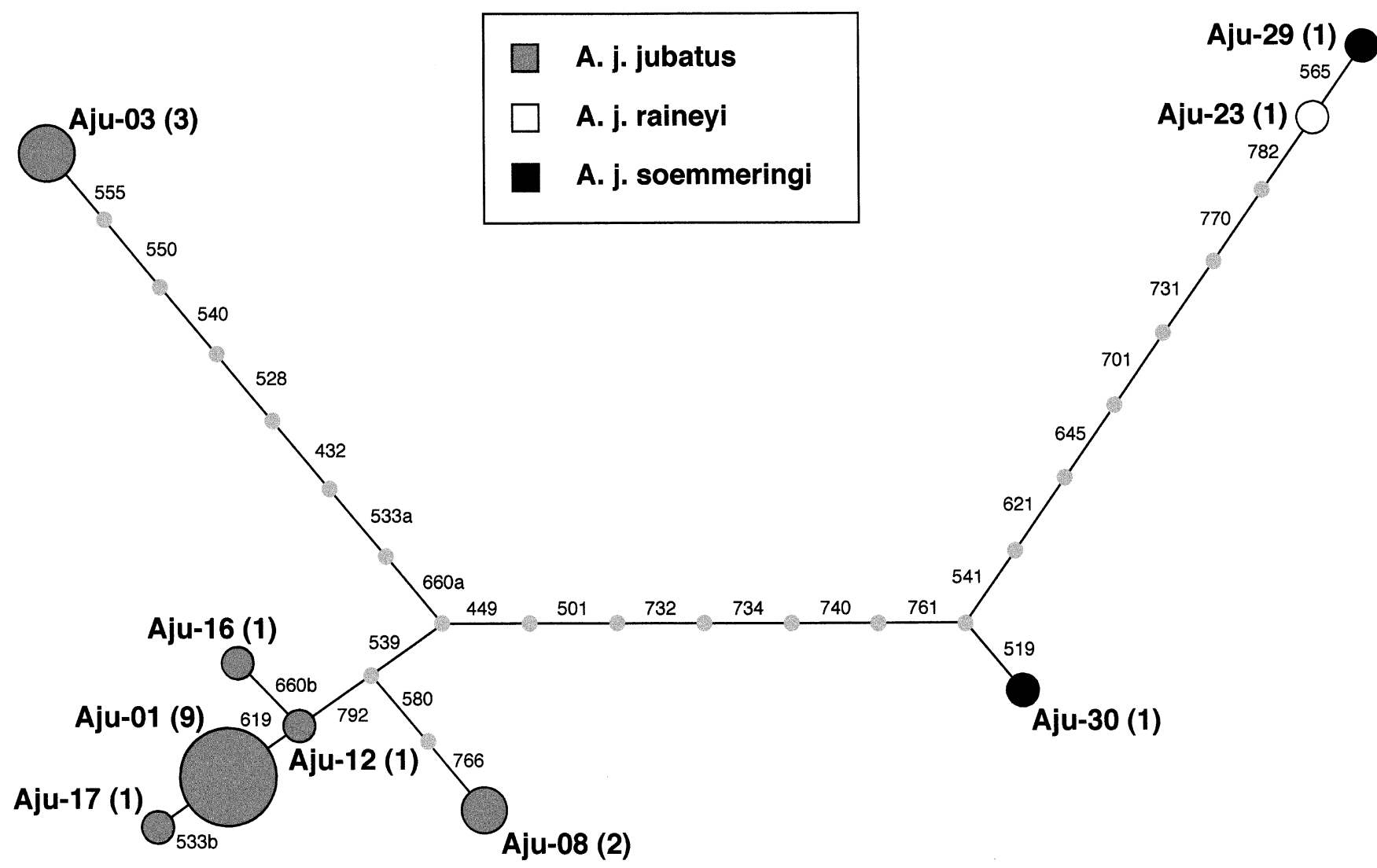

Fig. 2 Reduced median network of cheetah mtDNA control region segments. The area of the circles in the network is a measure of the sequences that are represented in the sphere and this figure is shown in parentheses beside the name of the sample. Sequences in the same circle are identical. The small grey circles represent other possible intermediate or ancestral haplotypes. The mutations that separate the mitotypes are numbered according to Fig. 1. The largest area represented on the network, Aju-01, may represent an ancestral haplotype from which the others evolved. 
To assess how this amount of variation compares to other members of the Felidae, the sequences produced were compared with control region sequences from another domestic cat and two South American cat species. A sample of the more complete ocelot (Leopardus pardalus) and margay (Leopardus wiedii) sequences analysed by Eizirik et al. (1998) was used. The available sequence that all of the species had in common was aligned and the average variation was calculated. The average nucleotide diversity in the 20 cheetah sequences was 0.0131 . This was approximately a third of the divergence between two domestic cat samples (0.0416). The average nucleotide diversities in the ocelots and margays were higher again at 0.0522 and 0.0745 , respectively. A neighbour-joining tree constructed using a 320-bp aligned portion of the sequences (Fig. 3) illustrates the differing amounts of variation displayed within the four cat species.

\section{Evolution of $R S 2$ repeated arrays in the cheetah control region}

The cheetah was found to contain the RS2 repetitive region that is also found in the domestic cat and many other species (Hoelzel et al., 1994). Each of the 20 individuals analysed was found to have an 80 -bp repeat unit that was repeated four times. The repeat units were defined using the consensus sequence from Wilkinson et al. (1997) as a guide and are denoted $\boldsymbol{a}, \boldsymbol{b}, \boldsymbol{c}$ and $\boldsymbol{d}$ as ordered on the mtDNA. Figure 4 shows an alignment of the different repeat units. Six different composite RS2 haplotypes were seen in the sample of cheetahs.

The evolution of the RS2 region was examined by treating each different repeat unit as an operational taxonomic unit (OTU) and constructing a phylogenetic tree (Fig. 5). Bootstrap values are based on 1000 trials. The neighbour-joining tree clearly shows that all of the $\boldsymbol{a}$ repeat units from the cheetah have grouped together. The same is true of all the $\boldsymbol{d}$ repeats which cluster on another branch of the tree and which are most distant from the $\boldsymbol{a}$ units. The two central repeat units, $\boldsymbol{b}$ and $\boldsymbol{c}$,

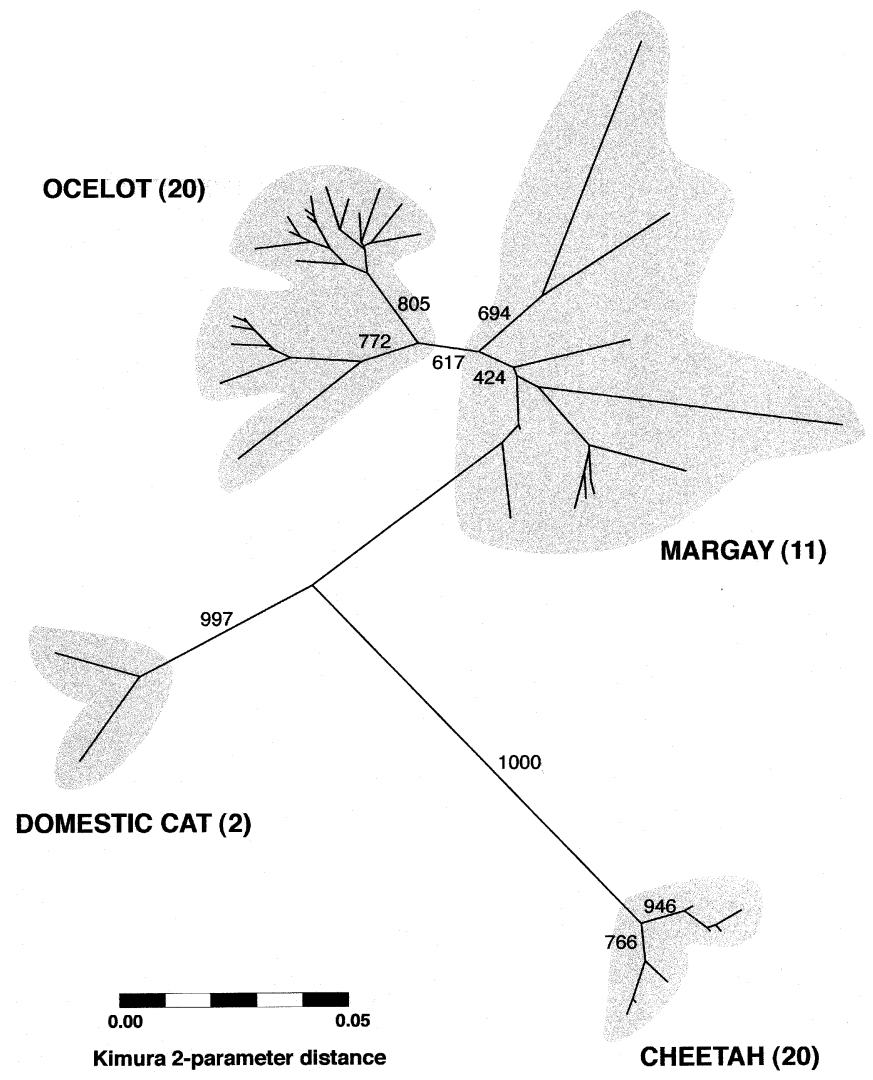

Fig. 3 Neighbour-joining tree for cheetah, domestic cat, ocelot and margay. The numbers in parentheses represents the number of sequences used for each species. Bootstrap values are indicated on the branches separating the major clusters of the tree. Positions with gaps were not excluded from the alignment as many of the informative sites in the cheetah would have been removed from the analysis.

are intermingled on the other branches of the tree. Based on this topology it is likely that concerted evolution is taking place between the two central repeats, $\boldsymbol{b}$ and $\boldsymbol{c}$, which is not affecting the two flanking elements.

MFOLD from the Wisconsin Package was use to test the cheetah and domestic cat repetitive sequences for putative secondary structure and similar patterns of

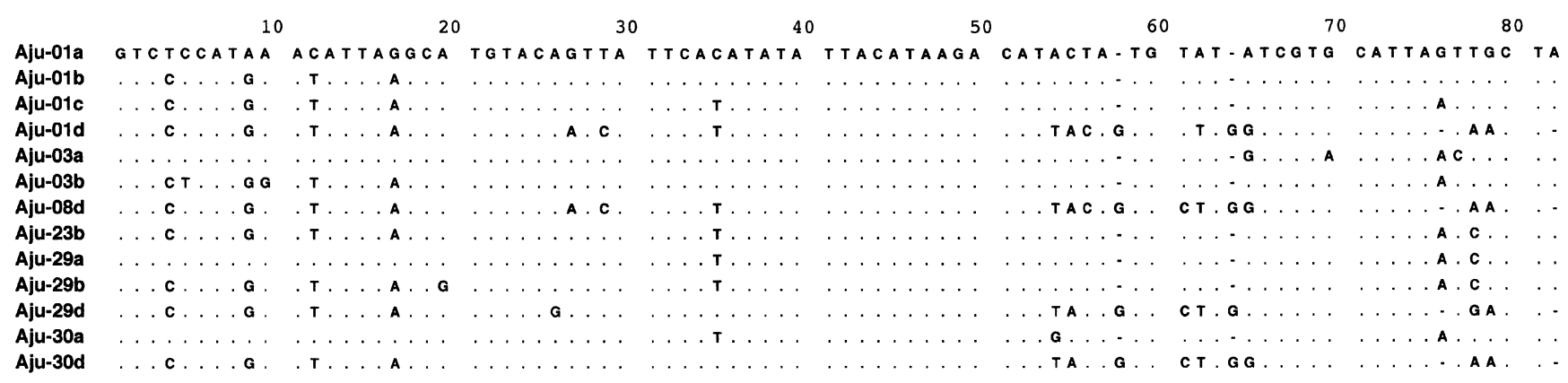

Fig. 4 Sequence alignment of different haplotypes at the cheetah RS2 region (beginning at 16478 in the Fca-01 sequence).

(C) The Genetics Society of Great Britain, Heredity, 86, 355-362. 


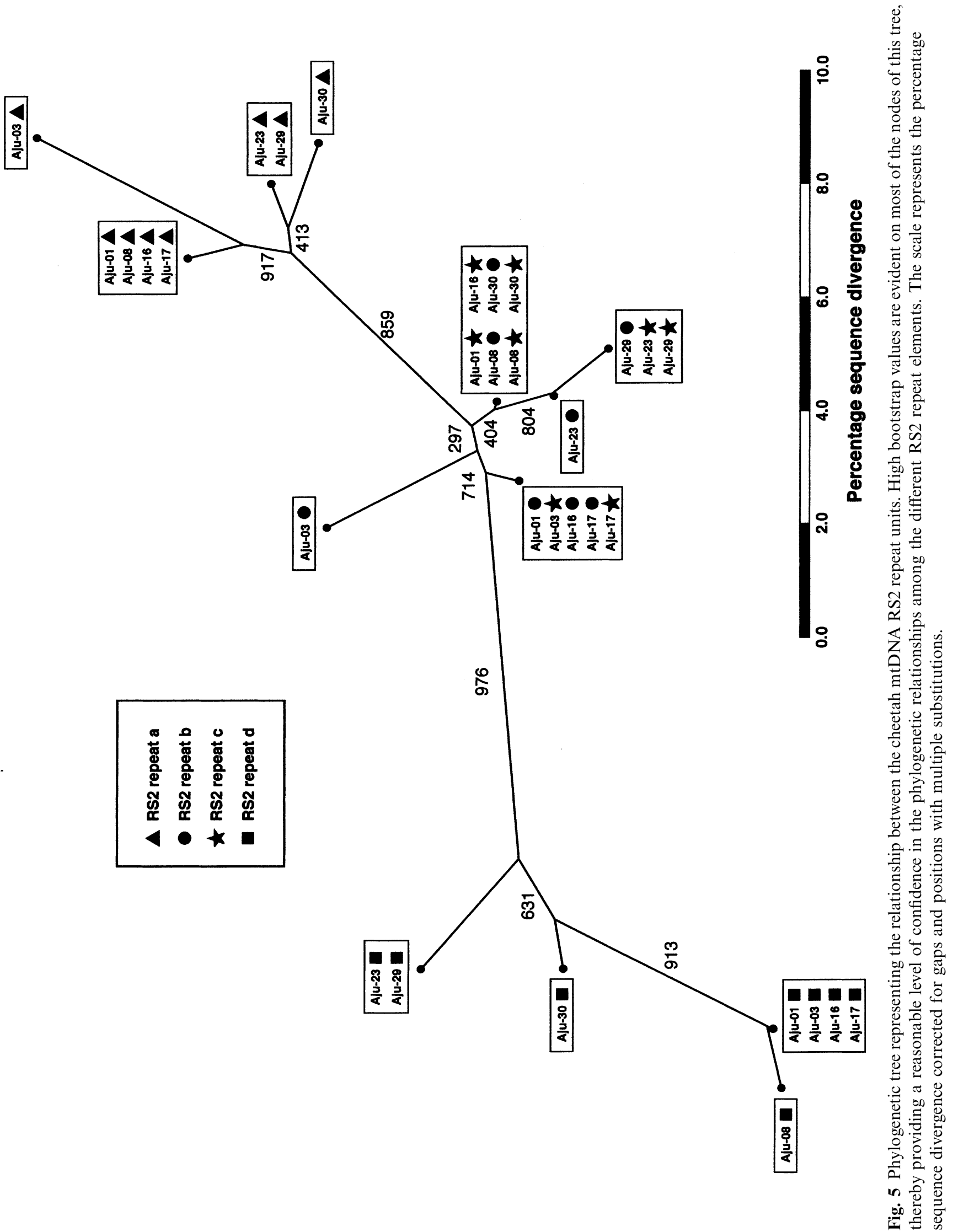

递

(c) The Genetics Society of Great Britain, Heredity, 86, 355-362. 
secondary structure were observed in each (results not shown). Over half of the sequence (51\% in cat and $56 \%$ in cheetah) is sequestered into base-paired regions.

\section{Discussion}

\section{Cheetah genetic variation}

The control region is the most rapidly evolving segment of the mitochondrial genome and the cheetah is the most widely quoted example of a genetically depauperate species. Any residual genetic variation in cheetahs should therefore be detectable using control region sequences. In the present study 525 base pairs were sequenced from 20 cheetahs and one domestic cat. Eight different haplotypes were detected. The four most distinct mitotypes differ from each other by at least eight mutations (Fig. 1). The network supports the subspecies division between northern and southern subspecies. The 17 A. j. jubatus Namibian samples group on the left-hand side of the network but are split into two distinct clades. The representatives from the northern subspecies are on the right-hand side of the network but do not group on the basis of their subspecies classification. The sequence of the raineyi sample and one of the soemmeringi individuals only differs by one substitution while the two soemmeringi individuals differ by nine substitutions.

Even though the cheetah samples came from different areas of Africa and incorporate representatives from three subspecies there is relatively little variation present in the 20 samples. Average nucleotide diversities estimated within species and the neighbour-joining tree in Fig. 3 demonstrate that they lack variation relative to two other wild species and the domestic cat and support previous assertions of relatively low levels of genetic variation in cheetah (O'Brien et al., 1983, 1987; Menotti-Raymond \& O'Brien, 1993).

The apparent genetic homogeneity of the two putative northern subspecies may have implications for those undertaking breeding programmes and maintaining captive populations. Interbreeding between the different subspecies may act to increase genetic variation in offspring (Menotti-Raymond \& O'Brien, 1993).

\section{RS2 region evolution}

Cheetah mitochondria contain the RS2 repeat region $(4 \times 80 \mathrm{bp})$ that has also been found in several other carnivore species. From the sequence alignment (Fig. 1) it can be seen that cheetahs and domestic cats show similarity at this region. Analysis of the cat repeats, using the program FOLD, revealed that the repeat unit may be capable of forming secondary structure (Lopez et al., 1996). MFOLD from the Wisconsin package revealed similar regions of secondary structure in the cheetah repetitive region. This situation was first reported in 1986, when it was observed that the control regions of rats could be arranged into cloverleaf secondary structures and it was hypothesized that these might be of functional importance (Brown et al., 1986). It has been speculated that this secondary structure may be associated with tandemly repeated arrays and replication control sites (Hoelzel et al., 1991; Sbisà et al., 1997).

Wilkinson et al. (1997) have identified two proteins that bind to the RS2 and which may cause DNA polymerase to stall, causing instability and increasing the likelihood of reiterative strand slippage (Weitzmann et al., 1997). The RS2 cloverleaf structures become more energetically stable as more repeat units are added to the array and may be further stabilized by a protein interaction. (Hoelzel et al., 1991; Fumagalli et al., 1996; Sbisà et al., 1997).

Nine repeats are present in the Florida panther (Puma concolor) mitochondria which suggests that further expansion of the repeat unit in other felids may occur. However, given that genomes with many repeats may be out-replicated by genomes with fewer repeats, their maintenance may be under positive selection (Wilkinson et al., 1997).

Figure 4 shows that the external repeat units in the RS2 array seem to have diverged, with the repeat closest to the centre of the control region being the most deviant. The two central repeats, $\boldsymbol{b}$ and $\boldsymbol{c}$, are most similar to each other and are intermingled on the branches on the tree. This is possibly a result of homogenization that may have been occurring between them. (Stewart \& Baker, 1994; Wilkinson et al., 1997). This may be explained by Buroker's model which predicts that the first and last copies of a repeat array will diverge and that the central repeats will evolve in a concerted manner (Buroker et al., 1990). A similar pattern of non-uniform evolution of repeat arrays in shrews and rabbits has also been reported (Stewart \& Baker, 1994; Casane et al., 1997).

\section{Acknowledgements}

We would like to acknowledge the assistance and advice of Kevin Glynn (Treanor and Glynn Veterinary Hospital, Dun Laoghaire, Co. Dublin), Neil Stronach (Fota Wildlife Park), Stephen Park, David Magee, Teresa Cymbron, Jill Bailey, Aoife McLysaght and two anonymous referees. A.R.F. is the recipient of a Wellcome Studentship in Biodiversity (grant no. 054275/Z/98/Z). D.E.M. is the recipient of a Wellcome Fellowship in Bioarchaeology (grant no. 047485/Z/96/Z). 
Sequences have been submitted to GenBank, Accession Numbers (AF348622-AF348642).

\section{References}

BANDELT, H. J., FORSTER, P., SYKES, B. C. AND RICHARDS, M. B. 1995. Mitochondrial portraits of human populations using median networks. Genetics, 141, 743-753.

BRown, G. G., GADAletA, G., PePE, G., SACCONE, C. ET AL. 1986. Structural conservation and variation in the D-loop-containing region of vertebrate mitochondrial DNA. J. Mol. Biol., 192, 503-511.

BUROKER, N. E., BROWN, J. R., GILBERT, T. A., O'HARA, P. J. ET AL. 1990. Length heteroplasmy of sturgeon mitochondrial DNA: an illegitimate elongation model. Genetics, 124, 157-163.

CASANE, D., DENNEBOUY, N., DE ROCHAMBEAU, H., MOUNOLOU, J. C. ET AL. 1997. Nonneutral evolution of tandem repeats in the mitochondrial DNA control region of lagomorphs. Mol. Biol. Evol., 14, 779-789.

CAUghley, G. 1994. Directions in conservation biology. J. Anim. Ecol., 63, 215-244.

EIZIRIK, E., BONATTO, S. L., JOHNSON, W. E., CRAWSHAW, P. G., JR ET AL. 1988. Phylogeographic patterns and evolution of the mitochondrial DNA control region in two neotropical cats (Mammalia, Felidae) J. Mol. Evol., 47, 613-624.

FELSENSTEIN, J. 1993. PHYLIP (Phylogeny inference package), version 3.527c. Distributed by the author, Department of Genetics, University of Washington, Seattle.

FUMAGAlli, L., TABERLET, P., FAVRE, L. AND HAUSSER, J. 1996. Origin and evolution of homologous repeated sequences in the mitochondrial DNA control region of shrews. Mol. Biol. Evol., 13, 31-46.

HOELzEL, A. R. 1993a. Evolution by DNA turnover in the control region of vertebrate mitochondrial DNA. Curr. Opin. Genet. Dev., 3, 891-895.

HOELZEL, A. R., HANCOCK, J. M. AND DOVER, G. A. 1993 b. Generation of VNTRs and heteroplasmy by sequence turnover in the mitochondrial control region of two elephant seal species. J. Mol. Evol., 37, 190-197.

HOElzEL, A. R., HANCOCK, J. M. AND DOVER, G. A. 1991. Evolution of the cetacean mitochondrial D-loop region. Mol. Biol. Evol., 8, 475-493.

HOELZEL, A. R., LOPEZ, J. V., DOVER, G. A. AND O’BRIEN, S. J. 1994. Rapid evolution of a heteroplasmic repetitive sequence in the mitochondrial DNA control region of carnivores. J. Mol. Evol., 39, 191-199.

KIMURA, M. 1980. A simple method for estimating evolutionary rates of base substitutions through comparative studies of nucleotide sequences. J. Mol. Evol., 16, 111-120.

LOPEZ, J. V., CEVARIo, S. AND O'BRIEN, S. J. 1996. Complete nucleotide sequences of the domestic cat (Felis catus) mitochondrial genome and a transposed mtDNA tandem repeat (Numt) in the nuclear genome. Genomics, 33, 229-246.

MENOTTI-RAYMOND, M. AND O'BRIEN, S. J. 1993. Dating the genetic bottleneck of the African cheetah. Proc. Natl. Acad. Sci. U.S.A., 90, 3172-3176.

MENOTTI-RAYMOND, M. AND O'BRIEN, S. J. 1995. Evolutionary conservation of ten microsatellite loci in four species of Felidae. J. Hered., 86, 319-322.

NOWELL, K. AND JACKSON, P. 1996. IUCN Wild Cats status survey and conservation action plan, pp. 12-44, Gland: Cambridge.

O'BRIEN, S. J., WILDT, D. E., GOLDMAN, D., MERRIL, C. R. ET AL. 1983. The cheetah is depauperate in genetic variation. Science, 221, 459-462.

O'BRIEN, S. J., ROELKE, M. E., MARKER, L., NEWMAN, A. ET $A L$. 1985. Genetic basis for species vulnerability in the cheetah. Science, 227, 1428-1434.

O'BRIEN, S. J., WILDT, D. E. AND BUSH, M. 1986. The cheetah in genetic peril. Sci. Am., 254, 68-76.

O'BRIEN, S. J., WILDT, D. E., BUSH, M., CARO, T. M. ET AL. 1987. East African cheetahs: evidence for two population bottlenecks? Proc. Natl. Acad. Sci. U.S.A., 84, 508-511.

SAMBROOK, J., FRITSCH, E. F. AND MANIATIS, T. 1989. Molecular Cloning: A Laboratory Manual. Cold Spring Harbor Laboratory, Cold Spring Harbor, NY.

SBISà, E., TANZARIEllo, F., ReYeS, A., PESOle, G. ET AL. 1997. Mammalian mitochondrial D-loop region structural analysis: identification of new conserved sequences and their functional and evolutionary implications Gene, 205, $125-140$.

SCHNEIDER, S., KEUFFER, J.-M., ROESSLI, D. AND EXCOFFIER, L. 1997. ARLEQUIN, version 1.1. A software package for population genetic data analysis. Genetics and Biometry Laboratory, University of Geneva, Switzerland.

SOLIGNAC, M., MONNEROT, M. AND MOUNLOU, J.-C. 1986. Concerted evolution of sequence repeats in Drosophila mitochondrial DNA. J. Mol. Evol., 24, 53-60.

STEWART, D. T. AND BAKER, A. J. 1994. Patterns of sequence variation in the mitochondrial D-loop region of shrews. Mol. Biol. Evol., 11, 9-21.

THOMPSON, J. D., HIGGINS, D. G. AND GIBSON, T. J. 1994. CLUSTAL $\mathrm{w}$ : improving the sensitivity of progressive multiple sequence alignment through sequence weighting, position-specific gap penalties and weight matrix choice. Nucl. Acids Res., 22, 4673-4680.

WAYNE, R. K., MODI, W. S. AND O'BRIEN, S. J. 1986. Morphological variability and asymmetry in the cheetah, a genetically uniform species Evolution, 40, 78-85.

WEITZMANN, M. N., WOODFORD, K. J. AND USIDIN, K. 1997. DNA secondary structures and the evolution of hypervariable tandem arrays. J. Biol. Chem., 272, 9517-9523.

WILKINSON, G. S., MAYER, F., KERTH, G. AND PETRI, B. 1997. Evolution of repeated sequence arrays in the D-loop region of bat mitochondrial DNA. Genetics, 146, 1035-1048. 